\title{
Phase-Sensitivity Analysis of Injection-Locked Mutually Coupled Oscillators
}

\author{
Mabel Pontón, Silvia Hernández, Almudena Suárez \\ Dpto. Ingeniería de Comunicaciones \\ University of Cantabria \\ Santander, Spain
}

\begin{abstract}
A system of two injection-locked mutually-coupled oscillators intended for sensing applications is analyzed with the aid of a semi-analytical formulation, based on realistic oscillator models, extracted from harmonic-balance simulations. Two different configurations are compared. In the first configuration, only one oscillator is connected to the synchronizing source, whereas the second oscillator is tuned by a capacitive element. Explicit expressions for the synchronization bandwidth and the phase sensitivity versus the tuning element are derived, which should be useful for an optimized design. The system stability properties and their dependence on the coupling-network elements and injection amplitude are also investigated. In the second configuration, the two oscillators are injection locked by the synchronizing source. Results are validated with costly harmonic-balance simulations at circuit level and with experimental measurements.
\end{abstract}

Keywords-Oscillator, injection locking, harmonic balance, stability

\section{INTRODUCTION}

The frequency variation of a free-running oscillator versus reactance changes has been used as a sensing mechanism in several previous works [1-2]. However, the oscillator freerunning frequency is affected by noise and parasitics. To cope with this problem, sensors based on injection-locked mutually coupled oscillators (ILMCO) have recently been proposed [34]. The two mutually coupled oscillators are synchronized by a low phase-noise input signal and their phase shift varies with the reactance in one of the oscillators, used as the sensing element.

In previous works [3-4], approximate analytical models of the ILMCO of Adler's type have been provided, which successfully describe the operation of the coupled system. However, the simplified models may fail to accurately predict the behavior of transistor-based oscillators, containing a number of lumped and distributed circuit elements. Here oscillator models extracted from harmonic balance (HB) simulations of the individual oscillator circuit(s) [5], in freerunning conditions, are used for a realistic analysis of the ILMCO. This is based on a semi-analytical formulation of the system, relying on the HB models [5]. The formulation allows the derivation of an explicit expression for the frequency bandwidth in which the oscillators are synchronized to the external source. It also allows obtaining a closed-form expression of the phase shift variation with the tuning element, which is the most relevant characteristic of the ILMCO. Such an expression should allow a thorough adjustment of the phase-shift sensitivity.
Two different configurations are compared. In the first one, only one of the oscillators is injection locked, whereas the other one contains the reactance that is used as a sensing element. In the second configuration, inspired by [3], the two oscillators are simultaneously injection-locked by the synchronizing source. Because the object is not the sensor application but the derivation of a useful simulation methodology, results will be validated with $\mathrm{HB}$ simulations of the whole system at circuit level and with measurements.

\section{INJECTION-LOCKED COUPLED- OSCILLATOR SYSTEM}

The proposed system of two injection-locked mutuallycoupled oscillators is shown in Fig. 1. The injection source with amplitude $E_{g}$ and frequency $\omega_{s}$ is connected to Osc. 1 [Fig. 1(a)] and the tuning element belongs to Osc. 2. This element is a capacitor connected to the transistor source terminal [Fig. 1(b)]. The two oscillators are coupled using a symmetric network, consisting of a transmission line bounded by two series resistors $R_{c}$. The outputs of the two oscillators, with the same oscillation frequency and phase-shift $\phi$, are connected to a mixer acting as a phase detector.

The oscillators in the mutually-coupled system will be described with admittance-type models extracted from HB. In free-running conditions, the current-to-voltage ratio of each oscillator is equal to zero at all the circuit nodes, and, in particular, at the node where each oscillator is later connected to the system (Fig. 1). When this connection takes place, the node admittance function $Y$ will undergo an increment with respect to its original zero value. Assuming small amplitude $E_{g}$ and a weak coupling strength, the function $Y$ can be expressed as a first-order Taylor series expansion about $Y=0$. The free-running solution of each oscillator circuit will be expressed in terms of the fundamental-frequency amplitude and oscillation frequency: $\left(V_{o 1}, \omega_{o 1}\right)$ and $\left(V_{o 1}, \omega_{o 1}\right)$. The derivatives are calculated through finite differences in commercial $\mathrm{HB}$, with the aid of an auxiliary generator (AG) [5], introduced in parallel at the same node where the oscillator is coupled to the system. Derivatives with respect to the voltage amplitude and oscillation frequency will be denoted as $Y_{v}, Y_{\omega}$, respectively. In the case of Osc. 1, derivatives with respect to the real and imaginary parts of the injection source $Y_{E g, r}, Y_{E g, i}$ should also be calculated. In the case of Osc. 2, a derivative with respect to the tuning parameter $Y_{\eta}$ should also be obtained. 


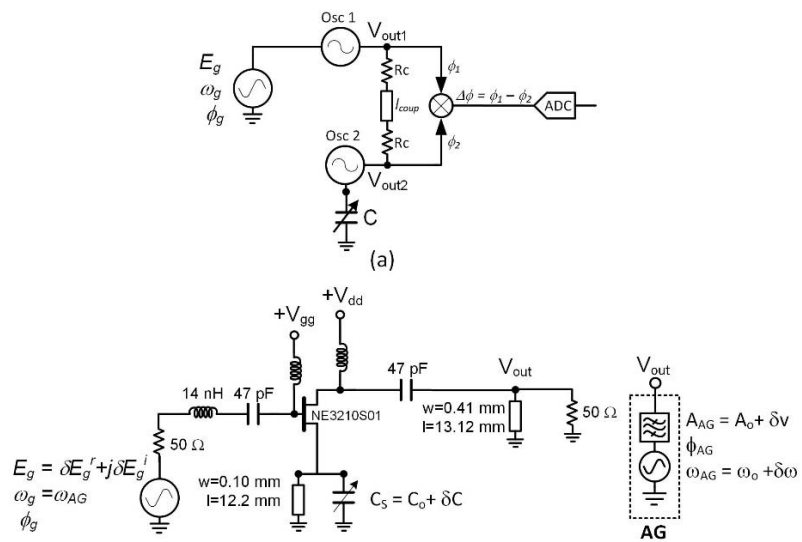

(b)

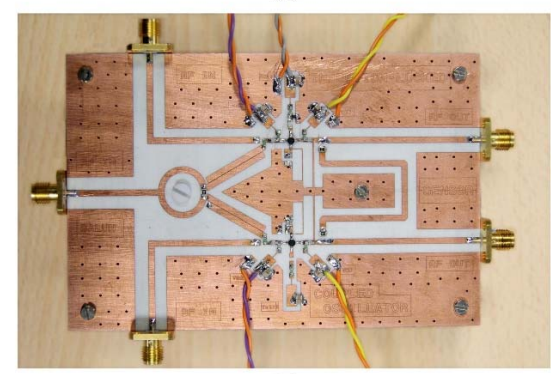

(c)

Fig. 1. (a) Proposed ILMCO topology. (b) Schematic of the individual oscillator based on the NE3210S01 FET transistor at $2.45 \mathrm{GHz}$. The AG used for the sequential calculation of the derivatives through finite differences is also represented. (c) Photograph of the prototype built on Rogers 4003C substrate.

In the most usual case, the two oscillator elements will be equal, with the same free-running solution $\left(V_{o}, \omega_{o}\right)$. Assuming also an injection-locked periodic, the coupled system is governed by the following complex equations:

$$
\begin{aligned}
& {\left[Y_{v} \Delta V_{1}+Y_{\omega} \Delta \omega_{s}\right]=-Y_{e}-Y_{n b} e^{j \phi}-Y_{E g, r} E_{g} \cos \phi_{g}-Y_{E g, i} E_{g} \sin \phi_{g}} \\
& {\left[Y_{v} \Delta V_{2}+Y_{\omega} \Delta \omega_{s}+Y_{\eta} \Delta \eta\right]=-Y_{e}-Y_{n b} e^{-j \phi}}
\end{aligned}
$$

where higher order terms have been neglected. The variables $\Delta V_{1}, \Delta V_{2}$ are the voltage-amplitude increments, $\Delta \omega_{s}$ is the frequency increment with respect to the free-running value $\omega_{o}$ and $\Delta \eta$ is increment in the tuning-parameter of Osc. 2. The system phase reference is taken at Osc. 1, which exhibits a phase shift $-\phi_{g}$ with respect to the injection source. In turn, $\phi$ is the phase shift between the two oscillator elements. On the other hand, $Y_{e}$ and $Y_{n b}$ are the element values of the admittance matrix of the symmetric coupling network, $Y_{e}=Y_{11}=Y_{22}$ and $Y_{n b}=Y_{12}=Y_{21}$. The formulation (1) is similar to the one developed in [5] for the case of coupledoscillator arrays with a number of oscillator elements $N \geq 3$. However, as will be shown, the qualitative behavior is different for $N=2$ and $N \geq 3$, due to the lack of symmetry in the tuning action. Splitting the complex equations in (1) into real and imaginary parts, one obtains:

$$
\begin{aligned}
& Y_{v}^{r} \Delta V_{1}+Y_{\omega}^{r} \Delta \omega=-Y_{e}^{r}-Y_{n b}^{r} \cos \phi+Y_{n b}^{i} \sin \phi \\
&-E_{g} Y_{E g r}^{r} \cos \phi_{g}-E_{g} Y_{E g i}^{r} \sin \phi_{g} \\
& Y_{v}^{i} \Delta V_{1}+Y_{\omega}^{i} \Delta \omega=-Y_{e}^{i}-Y_{n b}^{r} \sin \phi-Y_{n b}^{i} \cos \phi \\
&-E_{g} Y_{E g r}^{i} \cos \phi_{g}-E_{g} Y_{E g i}^{i} \sin \phi_{g} \\
& Y_{v}^{r} \Delta V_{2}+Y_{\omega}^{r} \Delta \omega+Y_{\eta}^{r} \Delta \eta=-Y_{e}^{r}-Y_{n b}^{r} \cos \phi-Y_{n b}^{i} \sin \phi \\
& Y_{v}^{i} \Delta V_{2}+Y_{\omega}^{i} \Delta \omega+Y_{\eta}^{i} \Delta \eta=-Y_{e}^{i}-Y_{n b}^{i} \cos \phi+Y_{n b}^{r} \sin \phi
\end{aligned}
$$

The increment $\Delta \omega_{s}$ is determined by the operation frequency of the injection source. However, $\Delta \omega_{s}$ must be contained in the locking bandwidth. Solving (2) for $\Delta \omega_{s}$, one obtains the following analytical expression:

$$
\Delta \omega_{s}=\left(Y_{v} \times Y_{e}+|A| \sin \phi+|B| \sin \phi_{g}\right) /\left(Y_{\omega} \times Y_{v}\right)
$$

where:

$$
\begin{aligned}
& A=\left(Y_{v} \times Y_{n b}\right)+j\left(Y_{n b} \cdot Y_{v}\right) \\
& B=E_{g}\left(Y_{v} \times Y_{E g, r}\right)+j E_{g}\left(Y_{v} \times Y_{E g, i}\right)
\end{aligned}
$$

The operators are $a \times b=a_{r} b_{i}-a_{i} a_{r}$ and $a \times b=a_{r} b_{i}-a_{i} a_{r}$, where the subindexes $r$ and $i$ indicate real and imaginary parts. For each amplitude $E_{g}$, the limits of the synchronization bandwidth such that phase shift $\phi$ can be varied in the full interval $\phi \in\left[-180^{\circ}, 180^{\circ}\right]$ are obtained by imposing magnitude one and opposite sign in the sinusoidal functions:

$$
\begin{aligned}
& \omega_{s, 1}=\omega_{o}-\frac{\bar{Y}_{v} \times \bar{Y}_{e}+|A|-|B|}{\left(\bar{Y}_{\omega} \times \bar{Y}_{v}\right)} \\
& \omega_{s, 2}=\omega_{o}-\frac{\bar{Y}_{v} \times \bar{Y}_{e}+|B|-|A|}{\left(\bar{Y}_{\omega} \times \bar{Y}_{v}\right)}
\end{aligned}
$$

Solving now (2) for $\Delta \eta$ and combining the resulting expression with (3), at each constant $\Delta \omega_{s}$, the relationship between $\Delta \eta$ and the phase-shift $\phi$ is:

$\Delta \eta=\frac{\left[Y_{n b} \cdot Y_{v} \sin \phi+\Delta \omega_{s}\left(Y_{\omega} \times Y_{v}\right)-Y_{v} \times Y_{e}-Y_{v} \times Y_{n b} \cos \phi\right]}{Y_{v} \times Y_{\eta}}$

Choosing the length of the transmission line in the coupling network so as to fulfill $Y_{v} \times Y_{n b}=0$ in (5), it is immediately derived that the maximum and minimum $\Delta \eta$ correspond to the phase-shift values: $-90^{\circ}$ and $90^{\circ}$. As demonstrated in the next section, the maximum stable phase interval is $\left(-90^{\circ}, 90^{\circ}\right)$, so under fulfillment of $Y_{v} \times Y_{n b}=0$ there is an univocal relationship between the tuning element and the phase shift. The offset of $\Delta \eta$ with respect to the free-running value can be eliminated by choosing the following injection frequency:

$$
\Delta \omega_{s}=\frac{Y_{v} \times Y_{e}}{Y_{\omega} \times Y_{v}}
$$

From inspection of (4), the above value belongs to the synchronization band. Then, the tuning parameter sensitivity can be expressed as: 


$$
\Delta \eta=\frac{Y_{n b} \cdot Y_{v}}{Y_{v} \times Y_{\eta}} \sin \phi
$$

As gathered from (7), the phase sensitivity increases when increasing the magnitude $\left|Y_{n b}\right|$.

The above expressions have been applied to the analysis of the ILMCO in Fig. 1. This is composed of two oscillators based on the FET transistor NE3210S01. The free-running frequency of each of the two oscillators is $f_{o}=2.45 \mathrm{GHz}$. Equation (6) provides $\Delta f_{s}=10 \mathrm{MHz}$ for weak coupling $\left(R_{c}=\right.$ $1 \mathrm{k} \Omega)$ and $\Delta f_{s}=32 \mathrm{MHz}$ for stronger coupling $\left(R_{c}=330 \Omega\right)$. The electrical length of the transmission line in the coupling network is chosen in each case so as to fulfill $Y_{v} \times Y_{n b}=0$. The resulting value is $354^{\circ}$ for $R_{c}=1 \mathrm{k} \Omega$ and $334^{\circ}$ for $R_{c}=330 \Omega$. Then, the variation of the phase-shift $\phi$ with the tuning element can be calculated using (5), which for $E_{g}=0.05 \mathrm{~V}$ and the two different $R_{c}$ values provides the curves in Fig. 2(a). Note that higher sensitivity is obtained for the weaker coupling strength since the complete phase-shift interval is obtained with a smaller variation of the tuning capacitor.
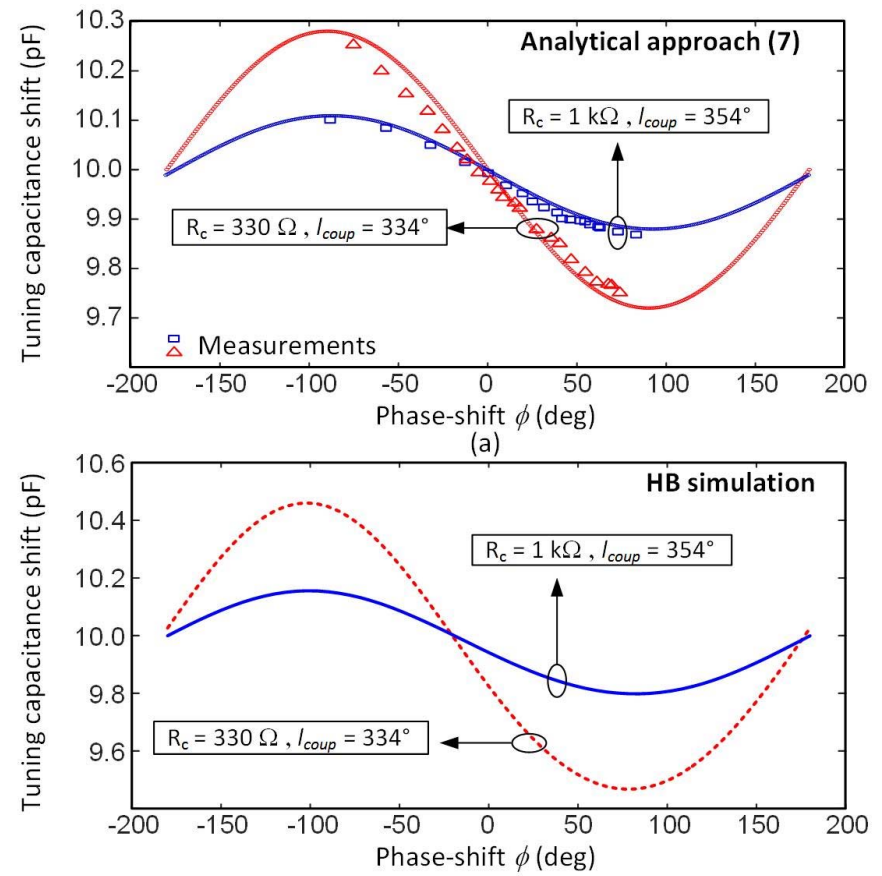

(b)

Fig. 2. Tuning parameter variation versus the phase-shift $\phi$ for $E_{g}=0.05 \mathrm{~V}$ (a) Using (7) with measurements superimposed. (b) $\mathrm{HB}$ circuit-level simulations.

In Fig. 2(b), the analytical results are compared with costly HB simulations of the entire system, at circuit level. Discrepancies are due to the fact that HB takes into account the coupling effects at all the harmonic terms. In contrast, the semi-analytical formulation only considers the coupling effects at the fundamental frequency, though the harmonic components are taken into account in the derivatives modeling the individual oscillators. Measurements have been superimposed in Fig. 2(a).

The configuration in Fig.1 has been compared with another one based on the recent work [3]. Unlike an I/Q excitation source, a $180^{\circ}$ phase-shift excitation source has been considered here, using a periodic signal generator and a balun. Now the derivatives used in the semi-analytical formulation are calculated in coupled conditions, though in a free-running regime, that is, under $E_{g}=0$. The system is governed by the following pair of complex equations:

$$
\begin{aligned}
& {\left[Y_{1, v 1} \Delta V_{1}+Y_{1, v 2} \Delta \bar{V}_{2}\right]=} \\
& -Y_{1, \omega} \Delta \omega-Y_{1, E g, r} E_{g} \cos \phi_{g}-Y_{1, E g, i} E_{g} \sin \phi_{g} \\
& {\left[Y_{2, v 1} \Delta V_{1}+Y_{2, v 2} \Delta \bar{V}_{2}+Y_{2, \eta} \Delta \eta\right]=} \\
& -Y_{2, \omega} \Delta \omega-Y_{2, E g, r} E_{g} \cos \phi_{g}-Y_{2, E g, i} E_{g} \sin \phi_{g}
\end{aligned}
$$

where $Y_{k, v k}, Y_{k, \omega}, Y_{k, E g, r}, \quad Y_{k, E g, i}, Y_{k, \eta}$ with $k=1,2$ are the admittance functions of the oscillator $k$ with respect to the voltage amplitude of oscillator $k$, frequency $\omega$, injection source $E_{g}$ and tuning parameter $\eta$, respectively, extracted about the system free-running point $E_{g}=0$, by means of two AGs, simultaneously connected to the system. On the other hand, $\Delta V_{1}$ is the increment in the voltage amplitude of Osc. 1 and $\Delta \bar{V}_{2}$ is the complex increment in the voltage of Osc. 2.

The relationship between $\Delta \eta$ and $\phi$ is obtained by splitting (8) into real and imaginary parts, sweeping the injectionsource phase $\phi_{g}$ and solving the resulting system for $\Delta \eta$ at each $\phi_{g}$ value. The phase-shift $\phi$ between oscillators elements can be obtained as $\phi=\arctan 2\left(V_{2 i} / V_{2 r}\right)$, where $V_{2 r}=V_{2 o r}+\Delta V_{2 r}$ and $V_{2 i}=V_{2 o i}+\Delta V_{2 i}$ and $V_{2 o r}, V_{2 o i}$ are the real and imaginary part of the second-oscillator voltage in free-running conditions. Fig. 3 shows the tuning parameter variation versus the phase-shift $\phi$ when solving system (8) for different values of $E_{g}$. The interval is centered about $180^{\circ}$ due to the use of the balun and increases with $E_{g}$. Measurements are superimposed.

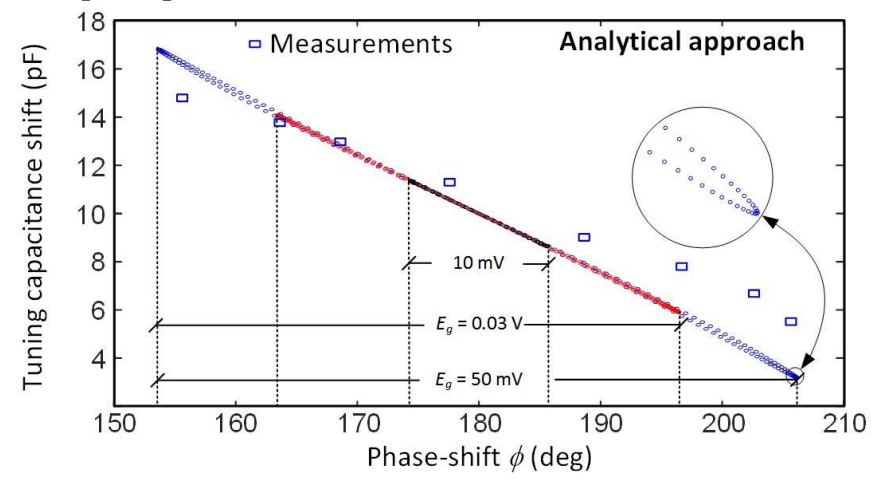

Fig. 3. Tuning parameter variation verus phase-shift derived from system (8) with $E_{g}=0.05 \mathrm{~V}$. Measurements are superimposed. 


\section{STABILITY ANALYSIS}

The stability of the ILMCO is evaluated by considering a small perturbation, which leads to a complex increment of the oscillation frequency, $s$, acting as time differentiator, as well as the following increments of the state variables:

$$
\begin{aligned}
& V_{1}(t)=V_{01}+\Delta V_{1}+\delta V_{1}(t), \\
& V_{2}(t)=V_{02}+\Delta V_{2}+\delta V_{2}(t), \\
& \phi_{1}(t)=0+\delta \phi_{1}(t), \\
& \phi_{2}(t)=\phi_{2}+\delta \phi_{2}(t),
\end{aligned}
$$

The perturbation of the first ILMCO configuration provides:

$$
\begin{gathered}
Y_{v}^{r} \Delta V_{1}+Y_{\omega}^{r} \Delta \dot{\phi}_{1}+Y_{\omega}^{i} \frac{\Delta \dot{V}_{1}}{V_{1}}=\frac{\partial F_{1}^{r}}{\partial \phi_{1}} \Delta \phi_{1}+\frac{\partial F_{1}^{r}}{\partial \phi_{2}} \Delta \phi_{2} \\
Y_{v}^{i} \Delta V_{1}+Y_{\omega}^{i} \Delta \dot{\phi}_{1}-Y_{\omega}^{r} \frac{\Delta \dot{V}_{1}}{V_{1}}=\frac{\partial F_{1}^{i}}{\partial \phi_{1}} \Delta \phi_{1}+\frac{\partial F_{1}^{i}}{\partial \phi_{2}} \Delta \phi_{2} \\
Y_{v}^{r} \Delta V_{2}+Y_{\omega}^{r} \Delta \dot{\phi}_{2}+Y_{\omega}^{i} \frac{\Delta \dot{V}_{2}}{V_{2}}=\frac{\partial F_{2}^{r}}{\partial \phi_{1}} \Delta \phi_{1}+\frac{\partial F_{2}^{r}}{\partial \phi_{2}} \Delta \phi_{2} \\
Y_{v}^{i} \Delta V_{2}+Y_{\omega}^{i} \Delta \dot{\phi}_{2}-Y_{\omega}^{r} \frac{\Delta \dot{V}_{2}}{V_{2}}=\frac{\partial F_{2}^{i}}{\partial \phi_{1}} \Delta \phi_{1}+\frac{\partial F_{2}^{i}}{\partial \phi_{2}} \Delta \phi_{2}
\end{gathered}
$$

where superindexes $r$ and $i$ indicate real and imaginary parts and the following functions have been defined:

$$
\begin{aligned}
& F_{1}=-Y_{e}-Y_{n b} e^{j\left(\phi_{2}-\phi_{1}\right)}-Y_{E g, r} E_{g} \cos \left(\phi_{g}-\phi_{1}\right)- \\
& Y_{E g, i} E_{g} \sin \left(\phi_{g}-\phi_{1}\right) \\
& F_{2}=-Y_{e}-Y_{n b} e^{j\left(\phi_{1}-\phi_{2}\right)}
\end{aligned}
$$

The phase derivatives in (10) must be particularized to each steady-state solution, given by $\phi_{1}=0, \phi_{2}=\phi$. System (10) can be expressed in matrix form as:

$$
\left[\begin{array}{c}
\Delta \dot{V}_{1} \\
\Delta \dot{V}_{2} \\
\Delta \dot{\phi}_{1} \\
\Delta \dot{\phi}_{2}
\end{array}\right]=[M]\left[\begin{array}{c}
\Delta V_{1} \\
\Delta V_{2} \\
\Delta \phi_{1} \\
\Delta \phi_{2}
\end{array}\right] ; \quad[M]=\left[M_{1}\right]^{-1}\left[M_{2}\right]
$$

where the matrixes $\left[\mathrm{M}_{1}\right]$ and $\left[\mathrm{M}_{2}\right]$ are directly obtained from the inspection of (11). The stability properties are determined by the eigenvalues of the matrix $[\mathrm{M}]$ in (12). From (3) two different $\phi_{g}$ functions are obtained for each $\Delta \omega_{s}$, though only one of them provides a stable $\phi$ interval, in consistency with the general behaviour of injection-locked oscillators [6]. The described eigenvalue analysis has been applied to the system in Fig. 1. In Fig. $4, R_{c}=1 \mathrm{k} \Omega$ and two different electrical lengths, $354^{\circ}$ (optimum) and $360^{\circ}$, have been considered. The value $354^{\circ}$ enables a symmetrical stable interval $\left(-90^{\circ}, 90^{\circ}\right)$. The value $360^{\circ}$ provides the stable interval $\left(-135^{\circ}, 45^{\circ}\right)$. When reducing the coupling resistor, the stability margin increases due to stronger coupling effects. The results of the stability analysis are consistent with the measurements presented in Fig. 2.

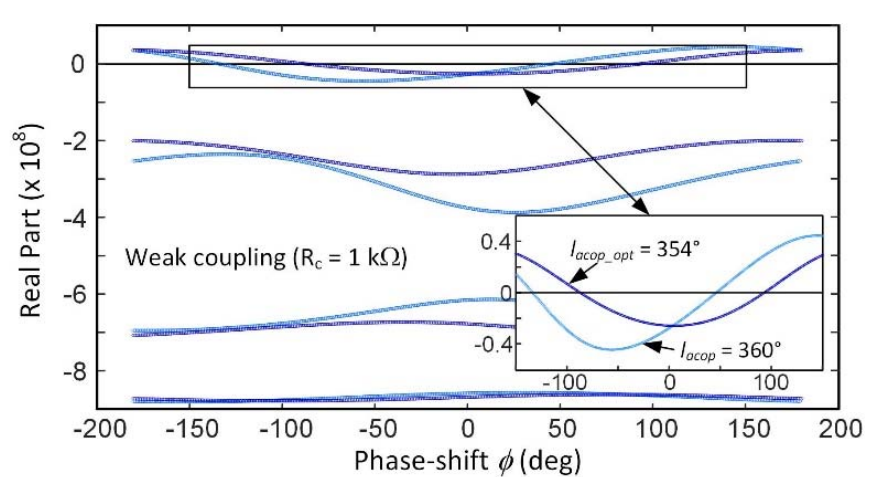

Fig. 4 Stability analysis of the ILMCO in Fig. 1 using (12). The results obtained with two different electrical lengths, $354^{\circ}$ (optimum) and $360^{\circ}$, are compared.

\section{CONCLUSION}

A system of two injection-locked mutually-coupled oscillators for possible application in sensors has been analyzed in detail. Analytical expressions for the synchronization bandwidth and the phase sensitivity with respect to the tuning element have been provided. Departing from these equations, a methodology to optimize the phase sensitivity has been obtained. The method has been validated with costly $\mathrm{HB}$ simulations at circuit level and with measurements.

\section{ACKNOWLEDGMENT}

The authors would like to thank to Spanish Ministry of Economy and Competitiveness for their financial support under the research project TEC2014-60283-C3-1-R and Juan de la Cierva Research Program IJCI-2014-19141 and by the Parliament of Cantabria under the project Cantabria Explora 12.JP02.64069.

\section{REFERENCES}

[1] H. Wang, C. C. Weng and A. Hajimiri, "Phase Noise and Fundamental Sensitivity of Oscillator-Based Reactance Sensors," IEEE Trans. Microw. Theory Techn, vol. 61, no. 5, pp. 2215-2229, May 2013.

[2] J. H. Oum, H. Koo and S. Hong, "Non-contact heartbeat sensor using LC oscillator circuit," 2008 30th Annual International Conference of the IEEE Engineering in Medicine and Biology Society, Vancouver, BC, 2008, pp. 4455-4458.

[3] J. C. Chien and A. M. Niknejad, "Oscillator-Based Reactance Sensors With Injection Locking for High-Throughput Flow Cytometry Using Microwave Dielectric Spectroscopy," IEEE J. Solid-State Circ., vol. 51, no. 2, pp. 457-472, Feb. 2016.

[4] J. Juillard, P. Prache and N. Barniol, "Analysis of Mutually InjectionLocked Oscillators for Differential Resonant Sensing," IEEE Trans. Circ. Systems I, vol. 63, no. 7, pp. 1055-1066, July 2016.

[5] A. Suárez, S. Sancho and F. Ramírez, "General Formulation for the Analysis of Injection-Locked Coupled-Oscillator Systems," IEEE Trans. Microw. Theory Techn., vol. 61, no. 12, pp. 4730-4744, Dec. 2013.

[6] K. Kurokawa, "Some basic characteristics of broadband negative resistance oscillators," Bell Syst. Tech. J., vol. 48, pp. 1937-1955, Jul.Aug., 1969. 